\title{
Upper-Division Students' Use of Separation of Variables
}

\author{
Steven J. Pollock and Bethany R. Wilcox \\ Department of Physics, University of Colorado, 390 UCB, Boulder, CO 80309
}

\begin{abstract}
Separation of variables can be a powerful technique for solving many of the partial differential equations that arise in physics contexts. Upper-division physics students encounter this technique in multiple topical areas including electrostatics and quantum mechanics. To better understand the difficulties students encounter when utilizing the separation of variables technique, we examined responses to midterm exam questions and conceptual assessments, and conducted think-aloud, problem-solving interviews. Our analysis was guided by an analytical framework that focuses on how students activate, construct, execute, and reflect on the separation of variables technique when solving physics problems. Here we focus on student difficulties with separation of variables as a technique to solve Laplace's equation in spherical coordinates in the context of junior-level electrostatics. Challenges include: recognizing when separation of variables is the appropriate tool, identifying implicit boundary conditions, and spontaneously reflecting on their solutions.
\end{abstract}

PACS numbers: 01.40.Fk

\section{INTRODUCTION}

A growing body of research on student learning at the upper-division level focuses on students' use of mathematics in physics problem solving (e.g., Refs [1-3]). For example, Partial Differential Equations (PDEs) appear in multiple contexts in the undergraduate physics curriculum (e.g., Laplace's equation and the Schrödinger equation). One of the most common approaches to solving PDEs is to turn them into multiple Ordinary Differential Equations (ODEs) using a technique known as separation of variables $(\mathrm{SoV})$. Here, we use the term SoV to refer to the technique of guessing a general solution with a functional form that allows the PDE to be separated into several ODEs and then solving these ODEs individually. This technique is not to be confused with the strategy (also conventionally referred to as separation of variables) used to solve separable ODEs.

At the University of Colorado Boulder (CU), physics students encounter SoV several times in their undergraduate courses. The first exposure is often in sophomore classical mechanics as a technique to solve Laplace's equation in Cartesian coordinates. It is also seen in junior electrostatics in the context of solving Laplace's equation for the electric potential in both Cartesian and azimuthally symmetric spherical coordinates. Spherical $\mathrm{SoV}$ is then seen again in quantum mechanics, this time without azimuthal symmetry where it is used to solve the Schrödinger equation for the hydrogen atom. However, despite these repeated exposures, we observe persistent student difficulties with the SoV technique, and some physics faculty at $\mathrm{CU}$ have expressed concern that students do not begin to demonstrate mastery of the SoV technique until they see it for a third time in quantum mechanics, and sometimes not even then.

Here, we focus on the use of SoV in spherical coordinates in junior-level electrostatics. Given the many uses of SoV, we do not claim that the issues identified here are exhaustive, but rather that they provide a sense of the kinds of challenges students face when dealing with
SoV. To help us structure our analysis of student work, we use the ACER framework. ACER is an analytical framework which organizes the problem solving process into four components: Activation of the mathematical tool, Construction of the model, Execution of the mathematics, and Reflection on the result [3]. ACER was designed to describe students' use of mathematical tools in physics problem solving, and is consistent with several other problem-solving frameworks $[4,5]$. The framework has been used previously to investigate student difficulties with integration [3] and the Dirac delta function [6].

\section{METHODS}

Data for this study were collected from the Electricity and Magnetism 1 (E\&M 1) at CU, which covers electrostatics and magnetostatics (Cht. 1-6 of Griffiths [7]). The student population is composed of physics and engineering physics majors with a typical class size of 30-60 students. At CU, E\&M 1 is often taught with varying degrees of interactivity through the use of research-based teaching practices including concept tests and tutorials.

We collected data from three sources: student solutions to instructor-designed questions on midterm exams ( $\mathrm{N}=509$ solutions), responses to the multiple-response Colorado Upper-division Electrostatics (CUE) Diagnostic [8] $(\mathrm{N}=145)$, and think-aloud student interviews $(\mathrm{N}=6)$. Exam data were collected from 11 exam questions spanning 9 semesters and taught by 7 different instructors. Interviewees were paid volunteers who responded to an email request for research participants. All interviewees had successfully completed E\&M 1 one to two semesters prior with course grades ranging from A-C. All prompts in this study provided an azimuthally symmetric boundary condition (BC) for the voltage on the surface of a spherical shell (e.g., $V(R, \theta)=V_{o} \cos ^{2}(\theta)$ ) and asked for the voltage inside and/or outside the shell. Variations on the prompts included: using different surface BCs (potentially in terms of Legendre polynomials), 
or only asking for the potential inside or outside. The CUE included a multiple-response version of this question which asked students to choose the easiest approach and to justify their choice (see [8] for prompt).

Exams were analyzed by coding each element of the operationalized ACER framework (below) that appeared in the student's solution. These elements were then further coded to identify fine-grained, emergent aspects of students' work. Interviews were also analyzed by classifying each of the students' major moves into one of the four components of the framework. As the CUE question was in a multiple-choice format, it provided quantitative data on the prevalence of certain difficulties.

\section{ACER \& SEPARATION OF VARIABLES}

One outcome of this research is the operationalization of the ACER framework for the use of SoV to solve the types of problems described earlier. Operationalization is the process of producing a researcher-guided outline of the key elements in a complete solution to the targeted problems. This outline is refined based on analysis of student work (see Ref. [3] for details). Element codes below are for labeling purposes only and do not imply a particular order, nor are all elements always necessary.

Activation of the tool: The first component of the framework involves identifying SoV as the appropriate mathematical technique to solve for the voltage. We identified three elements in the form of cues present in a prompt that may activate resources associated with SoV. A1: The question provides BCs and asks for SoV or provides the expression for the general solution

A2: The question provides BCs and uses language associated with SoV (e.g., Legendre polynomials)

A3: The question provides BCs and asks for the electric potential or voltage in a charge free region

We include element A1 as it is common for a prompt/exam to provide the general solution for the voltage in spherical geometries.

Construction of the model: This component deals with modifying the general expression for the solution to Laplace's equation so that it matches the BCs.

C1: Express all relevant BCs, both those explicitly given in the prompt/figure and those implicit from the physical situation (e.g., $V(r \rightarrow \infty) \rightarrow 0$ )

$\mathrm{C} 2$ : Apply each BC to the general solutions in order to solve for all unknown constants (set up only)

Note that these elements do not necessarily occur sequentially, either with respect to one another or with respect to elements of the Execution component.

Execution of the mathematics: This component of the framework deals with elements involved in executing the mathematical operations related to SoV.

E1: Calculate values for all unknown constants based on applying the BCs

E2: Manipulate algebraic expressions into forms that can be readily compiled and interpreted
Element E1 can be accomplished in a variety of ways often involving several smaller steps depending on the particular BCs (see Results). For Cartesian SoV, where students are often asked to derive and solve the ODEs resulting from SoV, there would be two additional elements of Execution relating to this process [9].

Reflection on the result: The final component includes elements related to checking and interpreting aspects of the solution, including intermediate steps and the final result. While many different techniques can be used to reflect on a physics problem, the following four are particularly common when dealing with SoV.

R1: Check the units of the final expression

R2: Check that the solution matches all BCs

R3: Check the solution's behavior in known limits

R4: Confirm that the solution satisfies Laplace's Eqn

Element R3 refers specifically to checking the functional dependence, rather than the value, of the voltage in known limits. For example, checking that $V(r \rightarrow \infty)=0$ would be considered R2 while showing that $V$ goes to zero as $1 / r$ would be R3. The final element of Reflection (R4) was added to the framework after initial analysis of student work where we observed that mistakes in the Construction and Execution components often resulted in solutions that did not satisfy Laplace's equation.

\section{RESULTS}

This section presents the analysis of common student difficulties with spherical SoV organized by component and element of the ACER framework.

Activation of the tool: Elements A1-A3 describe different types of prompts that can cue students to activate resources related to $\mathrm{SoV}$, loosely organized by likelihood that they will do so. Of the solutions to exam questions with implicit prompts (i.e., elements A2-A3), very few $(4 \%, \mathrm{~N}=16$ of 371$)$ utilized a method other than SoV (e.g., $\vec{E}=-\vec{\nabla} V(R, \theta))$. In contrast, on the multipleresponse CUE asked at the end of the semester just under half of our students (41\%, N=59 of 145) did not select $\mathrm{SoV}$ as the appropriate solution method. Common alternatives were Coulomb's law (25\%, N=15 of 59) and Gauss' law ( $49 \%, \mathrm{~N}=29$ of 59 ). This may be due to the fact that spherical SoV questions bear a superficial similarity to problems solvable via Coulomb's or Gauss' laws.

Interviews provided additional insight into Activation of spherical SoV. Of the six participants, three spontaneously suggested SoV as the correct solution method. The three remaining students only suggested using SoV after being reminded that Laplace's equation is a PDE. This result suggests that, as we might expect, the activation of SoV for these students was more closely linked to the formal mathematics of the problem, rather than the physical context. Students' overall success at Activation on exam questions seems to contradict the significantly lower success rate seen on the CUE and in interviews. 
TABLE I. Common difficulties with C2. Percentages are of just the students who struggled with $\mathrm{C} 2(\mathrm{~N}=90)$. Codes are not exhaustive or exclusive, thus the $\mathrm{N}$ need not sum to 90 .

\begin{tabular}{lcc}
\hline \hline Difficulty & N & Percent \\
\hline Not setting $r=R$ for the surface boundary & 21 & $23 \%$ \\
Problems with $P_{l}$ terms & 16 & $18 \%$ \\
$\quad$ e.g., dropping $P_{l}$ 's inappropriately & & \\
$\begin{array}{l}\text { Including both } A_{l} \text { 's and } B_{l} \text { 's in one expression } \\
\quad 10\end{array}$ & $11 \%$ \\
$\quad$ e.g., $\left(A_{l} R^{l}+\frac{B_{l}}{R^{l+1}}\right) P_{l}=V_{o} P_{l}$ & \\
Never applied the surface boundary condition & 18 & $20 \%$ \\
\hline \hline
\end{tabular}

One potential explanation for this is that students are pattern matching on the exams rather than internalizing a clear motivation for when/why SoV is appropriate. This interpretation is supported by the following comment made by one interview participant: "I guess I didn't understand this problem as well as I should have; I just remember going through a mathematical process to get it, and I knew that one really well." Moreover, both the timing of exams and the limited number of tractable SoV questions may encourage pattern matching.

Construction of the model: The Construction component deals with mapping between the physics and mathematics of a problem. For spherical SoV, this process includes identifying all necessary BCs (element C1), both those provided explicitly in the prompt and those that are implicit in the underlying physics of localized charges (e.g., $V(r \rightarrow 0) \neq \infty)$. Of the solutions that utilized SoV on the exams $(\mathrm{N}=488)$, almost two-thirds (61\%, N=298 of 488) included correct expressions for all explicit and implicit BCs. Of the remaining solutions, more than half $(62 \%, \mathrm{~N}=117$ of 190$)$ never expressed the relevant implicit BCs at $r=0$ and/or $r=\infty$. Despite this, the majority of these solutions $(89 \%, \mathrm{~N}=104$ of 117$)$ correctly eliminated either the $A_{l}$ (outside) or $B_{l}$ (inside) terms. This move was often accompanied by axiomatic statements like " $A_{l}$ 's go to zero outside." This finding is also consistent with the idea that some students are using pattern matching to guide their solution rather than clearly justifying their steps from the underlying physics.

Element C2 involves setting up equations to solve for all unknown constants in the general solution in order to match the BCs (note, solving these equations will be discussed in relation to the Execution component). Roughly a fifth of solutions (19\%, N=90 of 485) included issues with setting up the equations to solve for one or more constants. Common issues are documented in Table I.

In interviews, students tended to move quickly back and forth between identifying BCs and setting up equations to match them. For example, all five participants who solved the spherical SoV question began by identifying one of the two implicit BCs (element $\mathrm{C} 1$ ) and using it to correctly eliminate either the $A_{l}$ or $B_{l}$ terms (element $\mathrm{C} 2$ ). All of these students then moved on to matching the $\mathrm{BC}$ at the surface without commenting on either
TABLE II. Common difficulties with E1. Percentages are of just the students who struggled with $\mathrm{E} 1(\mathrm{~N}=125)$. Codes are not exhaustive or exclusive, thus the $\mathrm{N}$ need not sum to 125 .

\begin{tabular}{lcc}
\hline \hline Difficulty & N & Percent \\
\hline Off by a constant factor or sign & 47 & $38 \%$ \\
Incorrect term matching & 32 & $26 \%$ \\
$\quad$ e.g., keeping too many or not enough $P_{l}$ 's & & \\
Not finishing the calculation & 24 & $19 \%$ \\
\hline \hline
\end{tabular}

the second implicit $\mathrm{BC}$ or what region their expression would be valid for. When asked where their final expression was valid, all five interviewees initially argued it would be valid everywhere. Once they were specifically directed to consider limiting values of $r$, all interviewees recognized their solution was inconsistent with the remaining implicit BCs, but only one spontaneously considered the possibility of having separate expressions for $V(r)$ inside and outside the sphere. Thus, the interviews suggest that students' tendency to not spontaneously acknowledge some or all of the implicit BCs may discourage them from recognizing that their solution is valid only for certain regions of space or vice versa.

Execution of the mathematics: The Execution component deals with the procedural aspects of working through the mathematics of a physics problem. Once a student has used the BCs to set up expressions for the unknown constants (element C2), there are any number of mathematical manipulations that may be necessary to solve for these constants (element E1). We noted two common strategies that can be used in this process: (1) Fourier's trick - the strategy used to solve for the coefficients in a Fourier series via the integral properties of orthogonal functions; and (2) term matching - the strategy of exploiting the properties of orthogonal functions to directly match the coefficients of like terms. Of the exam solutions that showed explicit evidence of Execution ( $92 \%, \mathrm{~N}=469$ of 509$)$, the majority of solutions used term matching $(89 \%, \mathrm{~N}=405$ of 455$)$ to solve for the nonzero constants, while only a small fraction $(12 \%, \mathrm{~N}=56$ of 455) used Fourier's trick. This strong preference for term matching is appropriate and is likely a reflection of the fact that nearly all surface BCs given on exams at $\mathrm{CU}$ can be expressed as a sum of 1-3 Legendre polynomials.

When solving for the values of the unknown constants (element E1), roughly a quarter of students' solutions (27\%, N=125 of 469) contained various mathematical mistakes. Common issues are documented in Table II. The fraction of solutions with mathematical errors was higher in solutions that utilized Fourier's trick $(60 \%$, $\mathrm{N}=34$ of 56 ) than in solutions that utilized term matching $(21 \%, \mathrm{~N}=84$ of 405$)$. This is likely due, at least in part, to the fact that Fourier's trick represents an inherently more mathematically demanding strategy.

Element E2 deals with compiling all aspects of the solution into a single expression for the voltage. Roughly 
three-quarters of the solutions ( $73 \%, \mathrm{~N}=374$ of 509$)$ were completed enough to potentially include a final expression $V(\vec{r})$, and most $(83 \%, \mathrm{~N}=313$ of 374$)$ compiled one correctly (given their prior work). Common mistakes included not compiling a final expression $(23 \%, \mathrm{~N}=14$ of $61)$, dropping or adding terms $(25 \%, \mathrm{~N}=15$ of 61$)$, and not including the $r$-dependence from the general solution $(21 \%, \mathrm{~N}=13$ of 61$)$. Ultimately, only a small fraction of students $(8 \%, \mathrm{~N}=43$ of 509) had difficulties only with the Execution component (i.e., no earlier mistakes).

The interviews provided minimal insight into student difficulties in the Execution component, in part because only two of the five students made any mathematical errors. Both of these students initially failed to include the $r$-dependence from the general solution in their expression for the voltage. Comments made by these two students suggested that they were focusing on how their final expression matched the $\mathrm{BC}$ at $r=R$. As the $\mathrm{BC}$ does not have $r$-dependence, this may account for these students leaving the $r$-dependence out of their final expression. The overall success of the interviewees with respect to Execution may be due in part the fact that all of the interviewees used term matching rather than Fourier's trick to solve for the non-zero constants. Thus the mathematical manipulations required for this problem were minimal and purely algebraic. Overall, analysis of both the interviews and exam solutions suggest that Execution rarely represents the primary barrier to student success on spherical SoV problems.

Reflection on the Result: We identified four reflective checks that a student could use to gain confidence in (or detect problems with) their solution to problems involving spherical SoV. Only a small fraction of our students made explicit, spontaneous attempts to check their final expressions ( $8 \%, \mathrm{~N}=27$ of 360$)$ and the majority of these did so only by checking $\mathrm{BCs}(70 \%, \mathrm{~N}=19$ of 27$)$. It is possible that more of the exam students performed one of these reflective checks spontaneously, but did not explicitly write it down on their solution. However, Reflection was also less common in interviews, where only two of five students made spontaneous attempts to check their solution, by looking at units or BCs. One additional student suggested checking units after being asked how he might convince himself his solution was correct.

Two of the exam prompts directly targeted element R3 by asking students to comment on why they might expect the first term in the potential outside the sphere to behave as $1 / r$ (when $V(R, \theta)>0$ ). Of the solutions to these two exams, only a small fraction $(8 \%, \mathrm{~N}=6$ of 72$)$ articulated a fully correct argument. Similarly, all three of the interview students that were asked about limiting behavior of the potential needed explicit guidance to construct the correct argument. If a significant fraction of our students have difficulty producing an expectation for the behavior of $V$ at large $r$, this may contribute to why spontaneous checks of limiting behavior were so rare.

\section{CONCLUDING REMARKS}

We investigated upper-division student difficulties when using the SoV technique to solve Laplace's equation in the context of junior electrostatics by examining students' solutions to exam questions and think-aloud interviews. The ACER framework helped us to organize and categorize these difficulties within the problemsolving process. We found that our students encountered various issues when solving spherical SoV problems. Our students sometimes struggled to identify $\mathrm{SoV}$ as the appropriate mathematical technique. We also found that some students did not spontaneously identify all implicit $\mathrm{BCs}$ on the potential in spherical coordinates. Additionally, our students had an appropriate preference for term matching when solving for non-zero constants in spherical SoV problems. This tendency is likely a reflection of the nature of canonical spherical BCs. The difficulties identified in this paper represent a subset of students' difficulties with spherical SoV and may not include issues that might arise: in other student populations, from its uses in other coordinate systems, or in other contexts.

Given the concerns voiced by CU faculty, we found that students were more successful than anticipated when solving spherical SoV on exams. However, this level of success did not persist to the end-of-semester diagnostic or interviews. We hypothesize that this trend may be due to students pattern matching on the exams. Both the distinctive nature of the questions and the proximity of the exams to classroom instruction often make patternmatching a highly effective strategy for solving canonical spherical SoV problems. To address this, instructors might include homework and exam questions that minimize the potential effectiveness of pattern-matching (e.g., $V$ inside a spherical capacitor).

This work was funded by the NSF (CCLI Grant DUE1023028 and GRF under Grant No. DGE 1144083).
[1] T. I. Smith, J. R. Thompson, and D. B. Mountcastle, Phys. Rev. ST Phys. Educ. Res. 9, 020110 (2013).

[2] R. E. Pepper, S. V. Chasteen, S. J. Pollock, and K. K. Perkins, Phys. Rev. ST Phys. Educ. Res. 8, 010111 (2012).

[3] B. R. Wilcox, M. D. Caballero, D. A. Rehn, and S. J. Pollock, Phys. Rev. ST Phys. Educ. Res. 9, 020119 (2013).

[4] J. Docktor, Dissertation, University of Minnesota (2009).

[5] T. J. Bing, Dissertation, University of Maryland (2008).
[6] B. R. Wilcox and S. J. Pollock, Phys. Rev. ST Phys. Educ. Res. 11, 010108 (2015).

[7] D. J. Griffiths, Introduction to electrodynamics (Prentice Hall, 1999).

[8] B. R. Wilcox and S. J. Pollock, Phys. Rev. ST Phys. Educ. Res 10, 020124 (2014).

[9] B. Wilcox, Dissertation, University of Colorado Boulder (2015). 